\title{
A SEPARATOR-ANALYZER FLOWMETER FOR COMPLEX LIQUIDS IN MICROFLUIDIC SYSTEMS
}

\author{
Shramik Sengupta *, Babak Ziaie ${ }^{\#, *}$ and Victor H Barocas * \\ Departments of Biomedical Engineering* and Electrical and Computer Engineering \# \\ University of Minnesota (Twin Cities) \\ Minneapolis, MN 55455
}

\begin{abstract}
The measurement and control of the flow rate of fluids containing charged macro-molecular species (such as proteins) and particles is required for a number of applications, such as controlled drug delivery and micro-scale bioreactors. The flow rates that need to be measured for such systems can be as small as picoliters per second (microliters per day). We are developing a novel flow meter, called the separator-analyzer flow meter (or SAF meter), to measure the flow rate of suspensions laden with protein or other charged macromolecules or particles. The SAF meter consists of a channel that splits into two parts and then recombines. A pair of electrodes is positioned before the channel bifurcation. When a DC potential is applied across this pair of electrodes, the charged species in the mixture are dragged toward one electrode, and the solutions flowing into the two arms downstream differ in the concentration (separator). This difference in concentrations is inversely proportional to the flow rate of the original mixture. The concentration difference can be measured optically or electrochemically (analyzer). Based on the input to the separator (strength of the applied electric field), and the output from the analyzer (concentrations in the two arms), it is possible to obtain the flow rate of the solution or suspension (and consequently the amount of charged species). A milli-scale prototype has been fabricated and has been used to measure flow rates from $25-75 \mu \mathrm{l} /$ minute.
\end{abstract}

\section{INTRODUCTION}

In Biomedical Microsystems (Bio-MEMS), the fluid handled is typically a suspension of particles and macromolecules. (e.g., cell lysate, and protein or DNA solutions) [1],[2]. In many applications, the quantity of interest is often the mass-flow-rate of the solute. Besides, the flow rates that are typically encountered (micro-liters per day to microliters per second) imply that a different set of physical phenomena (diffusion, viscosity and electro-kinetic effects) become significant at these micrometer scales. This renders many of the techniques commonly used for flow sensing in the macro-scale [3] ineffective, and new techniques for measuring flow rates need to be developed. For any new flow sensor to be successful as an "on chip" device, it is also important that it be fabricated using the same techniques that are used for fabricating the rest of the system. Thus, building a flow sensor using standard micro-fabrication techniques would be a significant advance toward the development of an independent microfluidic system that incorporates pumps, flow sensors and circuit logic to implement feedback or optimal control strategies.
In this work, we present a new design for a flow sensor targeted to measure the flow rates of macromolecular solutions in channels. The geometry of this device is simple enough to allow fabrication using standard micro-fabrication processes. In addition, it takes into account (and makes use of) diffusion and electrophoresis, physical phenomena that become important at micrometer length scales.

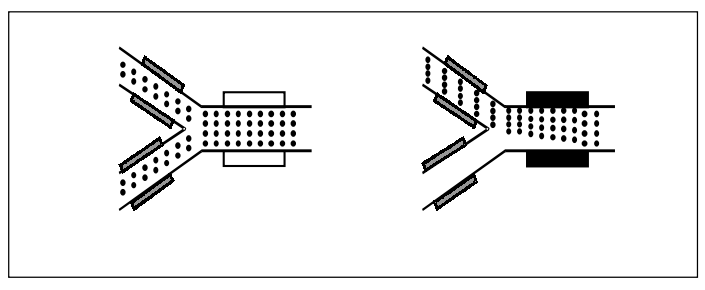

Figure 1: Schematic diagram showing the operating principle of the SAF meter. With no voltage applied to the separator (left panel) the concentration is unaffected. With a DC voltage to applied the separator (right panel) the charged species are steered.

\section{PRINCIPLE OF OPERATION}

Figure 1 schematically demonstrates the operating principle of the Separator-Analyzer Flow (SAF) meter. As seen in this figure, the flow sensing system consists of two parts, a main (undivided) channel and a pair of smaller channels. The smaller channels subsequently merge again (not shown in picture). A pair of electrodes is positioned across the channel just prior to the bifurcation. As long as no DC voltage is applied across these electrodes, the particles move with the fluid and are distributed equally between the two branch channels. However, if a constant DC voltage is applied, some of the particles in the fluid are dragged toward one electrode and the concentrations in the two fluid streams are different [Figure 1(right panel)]. If we assume plug flow for the fluid in the channel, then the difference in concentration is given by the equation

$$
\frac{\Delta C}{2 C_{0}}=\frac{\mu_{e} E L}{W}\left(\frac{1}{<V_{x}>}\right)
$$

where, $\Delta \mathrm{C}=$ Difference of the concentrations in the two branch arms, $\mathrm{C}_{0}=$ Concentration in the main channel,

$\mu_{\mathrm{e}}=$ Electrophoretic mobility of the particles,

$<\mathrm{V}_{\mathrm{x}}>=$ Average velocity of the fluid in the main channel,

$\mathrm{L}=$ Length of the separator electrodes, and

$\mathrm{W}=$ Half-width of the separator channel.

Travel support has been generously provided by the Transducers Research Foundation \& the DARPA MEMS \& DARPA BioFlips Program. 
We refer to this mode of operation of the sensor as the Constant Field Mode. It may be noted that there is a certain minimum velocity that can be measured. If the velocity is too low (or the field is too strong), all the particles are dragged down to the lower arm. Thus, another way to operate this device would be to control the electric field in such a way that the critical electric field (needed to keep all the particles in the lower arm) can be measured. In this case, the critical electric field $\left(\mathrm{E}_{\mathrm{c}}\right)$ is given by the relation

$$
E_{C}=\left(\frac{W}{L \mu_{e}}\right)<V_{x}>
$$

As evident from equations (1) and (2), the ability to measure flow is contingent upon the ability to measure the concentration of the particles in the suspension (or at the very least being able to distinguish between a suspension and a "clear" solvent). This can be done using various analytical techniques that can be broadly divided into two groups, optical and electrical. Measurement of absorbance could be a suitable optical technique for the analyzer since the absorbance of a protein solution at $280 \mathrm{~nm}$ is proportional to the concentration of the protein in the solution. [4]. This approach would be well suited to a static array of devices that could be scanned by a mounted laser, for example; but measuring absorbance "on chip" would be a considerable challenge. In addition, it is known that differences in the concentration of proteins in solution will cause the solutions to exhibit different dielectric constants $\left(\varepsilon_{\mathrm{r}}\right)$ [5], providing another potential concentration measurement tool. Electrodes placed across the solutions flowing through the two arms of the analyzer will then act like the plates of a parallelplate capacitor, whose capacitance $(\mathrm{C})$ is given by

$\mathrm{C}=\varepsilon_{0} \varepsilon_{\mathrm{r}}(\mathrm{A} / \mathrm{d})$

where, $\varepsilon_{0}$ is the permitivity of free space, $A$ is the surface area of the analyzer electrode and $d$ is the distance between the two electrodes. Thus, capacitance measurements may also be used in the analyzer. It may be noted that while the separator electrodes must be placed along the sidewalls of the channels, the analyzer electrodes can be placed either along the sidewalls or on the top and bottom of the channel.

\section{DESIGN ISSUES}

The purpose of the design exercise for this device is to specify a range of channel and electrode dimensions over which it will function and provide the corresponding minimum and maximum flow rates that can be measured. If operated in the constant field mode, the minimum measurable velocity is obtained when $\Delta \mathrm{C} / 2 \mathrm{C}_{\mathrm{o}}=1$ (corresponding to all the particles in the lower arm). Thus,

$$
<\mathrm{Vx}>_{\min }=\left(\mu_{\mathrm{e}} \mathrm{L} / \mathrm{W}\right)
$$

While the electrostatic force supplied by the DC field tries to bring all the particles toward one side of the channel, diffusion tries to homogenize the solution across the width of the channel. If we set the characteristic time for diffusion $\left(=\mathrm{W}^{2} / 2 \mathrm{D}\right.$; where $\mathrm{D}$ is the diffusivity of the particle in the solution) to be ten times larger than the characteristic time for electrophoretic migration $\left(=\mathrm{w} / \mu_{\mathrm{e}} \mathrm{E}\right)$, the least measurable flow rate $\left(\mathrm{Q}_{\min }\right)$ is given by the relation
$Q_{\min }=40 \mathrm{DL}(\mathrm{h} / \mathrm{W})$

where $\mathrm{h}$ is the height of the channel. We would ideally like $Q_{\min }$ to be as small as possible. Of the quantities in the above expression $D$ (the diffusivity of the protein) is beyond our control. The ratio of the channel height by width is determined by the etching technology used, (h/W) ratios of $\sim 1$ or lower can be obtained using isotropic etchants like $\mathrm{HF} / \mathrm{HNO}_{3}$, whereas more specialized anisotropic etching techniques will be required to obtain significantly higher values. The limit to how small the length of the electrode (L) can get is determined by the minimum feature size that can be drawn on the photolithography mask ( 5 microns in our case). Figure 2 shows how the minimum measurable flow rate varies for a solution of hen egg white lysozyme $\left(\mathrm{D}=7.5 \times 10^{-7} \mathrm{~cm}^{2} / \mathrm{s}[6]\right)$ as a function of these two sets of parameters.

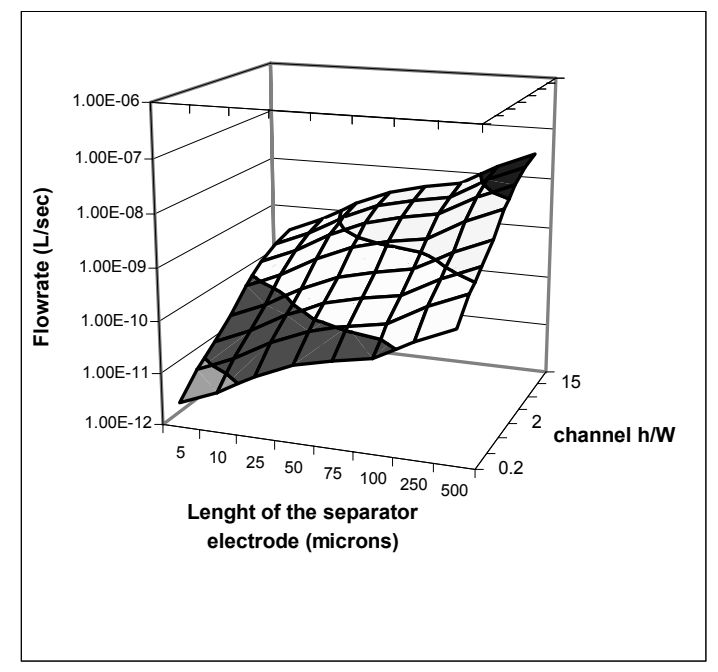

Figure 2: Plot of the minimum measurable flow-rate for a solution of lysozyme as a function of the channel and electrode parameters.

Once the hardware parameters have been chosen based on the minimum measurable flow rate, raising the field strength causes the surface plot in figure 2 would be shifted upward, thus allowing for the measurement of higher flow rates. The resolution of the measurement of the flow rate is a function of the resolution of the measurement of the concentration difference in the analyzer. As indicated by Figure 2, to measure a low flow rate, one would prefer a channel that is shallow but wide. However, to get a better sensitivity out of our (capacitive) analyzer, one would like the plates to be wide and distance between them to be low (see equation 3). Hence, we would prefer to place the electrodes of the analyzer on the top and bottom of the channels.

\section{MILLISCALE PROTOTYPE}

Although the goal of our work is to fabricate a micro-scale flow sensor capable of measuring flow rates as low as pico-liters per second (micro-liters per day), in order to verify the novel principle of operation experimentally, we first made a milli-scale prototype capable of measuring higher flow rates (of the order of micro-liters per hour) using the same principle. A picture of the prototype is shown in Figure 3. It was made out of commercially available UVcurable epoxy on top of a glass wafer. The top surface of the device 
is made out of Plexiglas, with holes drilled in for fluidic inlets and outlets. As seen in the picture, it consists of a main channel ( $1 \mathrm{~mm}$ wide) that splits into two branches $(500 \mu \mathrm{m}$ wide). All channels are 250 micron deep. The strip of platinum placed just prior to the bifurcation is 250 microns thick, and $1 \mathrm{~mm}$ wide. Thus, the effective length of the separator electrode is $1 \mathrm{~mm}$. The channels do not recombine, nor is there analyzer built into the device. However, the fluid in the two arms may be collected for off-line analysis.

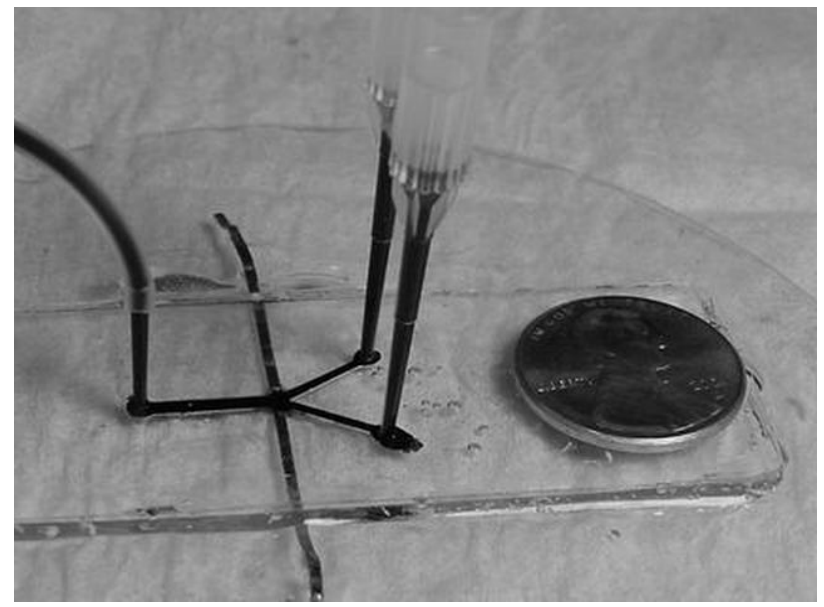

Figure 3: Picture of the milli-scale prototype used for conducting proof-of-concept experiments.

\section{PROOF-OF-CONCEPT EXPERIMENTS}

A set of experiments was conducted using the prototype shown above to experimentally verify the principle of operation of the SAF meter. The experimental protocol used is schematically illustrated in Figure 4.

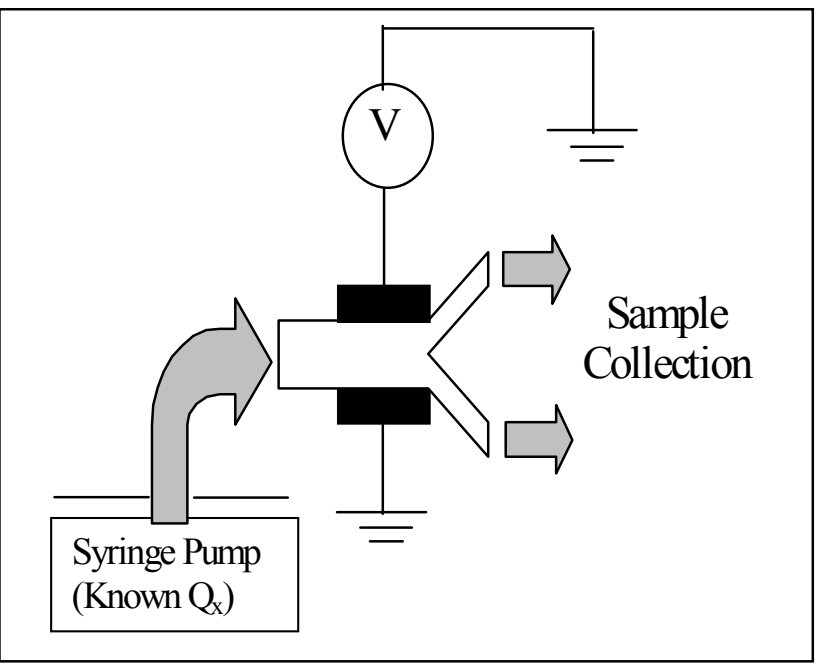

Figure 4: Schematic representation of the experimental protocol used to conduct proof-of-concept experiments.

As seen in the figure, we use a syringe pump to deliver known flow rates of a standard solution of Bovine Serum Albumin (BSA) in DI water. At the same time, a known DC potential difference is applied across the electrodes, and the fluid coming out of the two channels are collected. The concentration of BSA in the collected samples is measured by measuring the optical density (OD) of suitable dilutions of these samples at $280 \mathrm{~nm}$. The degree of separation $\left(\Delta \mathrm{C} / 2 \mathrm{C}_{0}\right)$ can be obtained from the measured concentrations.

\section{RESULTS}

Table 1 (below) summarizes our results for a set of imposed flow rates and applied voltages. It reports the mean (and standard deviation, $\mathrm{n}=3$ to 5 ) values of the degree of separation calculated from individual sets of observed concentrations. It may also be noted that only those observations were used for which the mass balance closed to within $\pm 5 \%$.

\begin{tabular}{|l|l|l|l|}
\hline & $25 \mu \mathrm{l} / \mathrm{min}$ & $50 \mu \mathrm{l} / \mathrm{min}$ & $75 \mu \mathrm{l} / \mathrm{min}$ \\
\hline 5 Volts & 0.1033 & 0.0689 & 0.0479 \\
& $(0.020)^{\star}$ & $(0.003)$ & $(0.007)^{\star}$ \\
\hline 10 Volts & 0.1986 & 0.1077 & 0.0838 \\
& $(0.027)$ & $(0.011)$ & $(0.020)$ \\
\hline 15 Volts & 0.3399 & 0.1236 & 0.1124 \\
& $(0.004)$ & $(0.019)^{\star}$ & $(0.015)^{\star}$ \\
\hline
\end{tabular}

Table 1: Observed values of $\left(\Delta C / 2 C_{0}\right)$ as a function of known values of flow rate $(Q)$ and applied voltage $(V)\left[^{*}\right.$ indicates the mean and standard deviation of five observations. (as opposed to that of three, for other cases)]

Equation (1) may be rewritten in terms of the applied voltage and imposed flow rate, as opposed to the electric field strength and the average velocity, respectively. On doing this, equation (1) assumes the form

$\frac{\Delta C}{2 C_{0}}=\frac{\mu_{e} L H}{W}\left(\frac{V}{Q}\right)$

Thus, if the obtained values of $\left(\Delta \mathrm{C} / 2 \mathrm{C}_{0}\right)$ are plotted against the ratio of the applied voltage and imposed flow rate, then the points must lie on a straight line, the slope of which will be a function of the dimensions of the channel and the electrode and the electrophoretic mobility of the protein. In Figure 5, we plot the (mean) values shown in Table1 against the ratio of the applied voltages and imposed flow rates. A straight line is then fitted through the points, and the equation of the straight line (along with the $\mathrm{R}^{2}$ value of the fit) is also displayed in the figure.

The high value for the $\mathrm{R}^{2}$ indicates that the experiments are in accordance with the theory. Also, equation (6) predicts that the slope of the line should be equal to $\left(\mu_{e} L H / W\right)$. Using the known values of the dimensions of the channel and the electrode, and the numerical value of the slope obtained from the fit, one may indirectly measure the electrophoretic mobility of the protein (equivalent to our flow measurement strategy). Performing the above exercise with the current data yields a value of $-17.8 \times 10^{-5} \mathrm{~cm}^{2} \mathrm{~V}^{-1} \mathrm{~s}^{-1}$ for the electrophoretic mobility of the protein (BSA) in DI water at a $\mathrm{pH}$ of 7. (The negative sign indicates that the protein has a net negative charge at that $\mathrm{pH}$.) The corresponding value in literature [7] was found to be $-12 \times 10^{-5} \mathrm{~cm}^{2} \mathrm{~V}^{-1} \mathrm{~s}^{-1}$. Thus our mathematical model, while qualitatively valid, seems to be lacking in the ability to 
correctly predict the degree of separation based on the flow rate (or vice-versa). Although tolerances in the dimensions of the separator would have contributed to the observed error, we believe that the main source of error lies in the primitive fluid flow model that we used and the assumption of a uniform electric field.

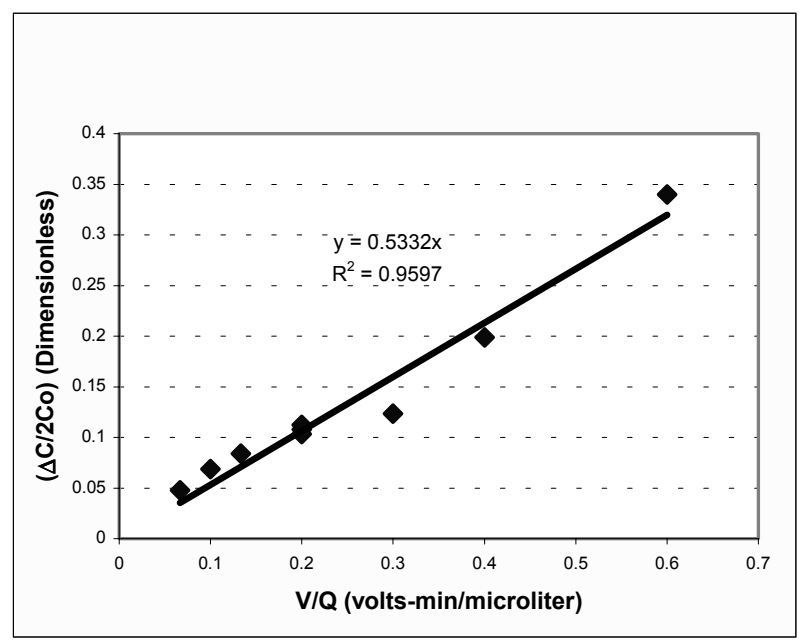

Figure 5:Plot of $\left(\Delta C / 2 C_{0}\right)$ vs. $(V / Q)$

\section{CONCLUSIONS}

In this work, we have proposed a novel method of measuring the flow rates of charged suspensions. The basic idea, i.e. that the degree of separation achieved by electrophoresis of the dissolved or suspended charged species in a direction perpendicular to the fluid flow could be used as a predictor for the flow rate of the fluid, was borne out by experiments conducted on a milli-scale prototype. Further, it may be noted that the SAF meter contains no moving parts, and that it specifically takes into account physical phenomena predominant at the micro-scale (such as electrophoresis and diffusion). These features make it ideal for incorporation into micro-fluidic systems. Besides, should the need arise, the SAF meter may also be calibrated as a mass-flow meter (for the suspended/dissolved species) since we measure concentrations to calculate the flow rate.

\section{ONGOING AND FUTURE WORK}

As may be obvious from the data presented, our experiments thus far have covered only a part of the possible values for the degree of separation (viz. from zero to one) that can be effected by the separator. We are currently conducting experiments similar to the ones described (but with different flow-rates and applied voltages) to make our demonstration more complete. Our technique at present also lacks an inbuilt (on-line) analyzer. We are currently building a milli-scale prototype of the analyzer to demonstrate the ability to measure protein concentrations using electrical techniques. Once this is done, smaller versions of the SAF meter (capable of measuring lower flow-rates) will be (micro)fabricated. The sensors thus fabricated will also have to be tested and characterized.

\section{Acknowledgements}

This work has been supported by a University of Minnesota Graduate School Grant in Aid (to VHB). We also gratefully acknowledge guidance received from Dr. Antonio Baldi and experimental help being received from Brett Hautala and Sarah Setiawan.

\section{REFERENCES}

1. D.L.Polla et al., "Microdevices in Medicine", Annual Reviews of Biomedical Engineering, 2 (2000), pp. 571-576

2. I. Walther, B. van der Schoot, M. Boillat, O Muller, and A Cogoli, "Microtechnology in space bioreactors", Chimia, 53,3 (1999) pp 7580

3 N. Cheremisinoff, Applied Fluid Flow Measurement, M. Decker, New-York, 1979.

4 F.M. Ausubel et al. Current Protocols in Molecular Biology, John Wiley and Sons, 2001

5. N. Nandi and B. Bagchi, "Anomalous dielectric relaxation of aqueous protein solutions", The Journal of Physical Chemistry A, 102, 43, (1998), pp. 8217-82221

6. S. Beretta, G. Ghirico, and G. Baldini, "Short-range interactions of globular proteins at high ionic strength", Macromolecules 33, 23, (2000) pp 8663-70.

7. N.G. Douglas, A.A. Humffray, H.R.C. Pratt, and G.W. Stevens, "Electrophoretic Mobility of proteins and protein mixtures" Chemical Engineering Science 50, 5 (1995) pp.743-754 